\title{
Coupled Cognitive and Functional Change in Alzheimer's Disease and the Influence of Depressive Symptoms
}

\author{
Laura B. Zahodne ${ }^{\mathrm{a}}$, D.P. Devanand ${ }^{\mathrm{b}}$ and Yaakov Stern ${ }^{\mathrm{a}, *}$ \\ ${ }^{a}$ Cognitive Neuroscience Division, Department of Neurology and Taub Institute for Research on Alzheimer's \\ Disease and The Aging Brain, College of Physicians and Surgeons, Columbia University, New York, NY, USA \\ ${ }^{\mathrm{b}}$ Department of Psychiatry, College of Physicians and Surgeons, Columbia University, New York, NY, USA
}

Handling Associate Editor: Josep Garre-Olmo

Accepted 10 December 2012

\begin{abstract}
In Alzheimer's disease (AD), cognition and function are only moderately correlated in cross-sectional studies, and studies of their longitudinal association are less common. One potential non-cognitive contributor to function is depression, which has been associated with poorer clinical outcomes. The current study investigated longitudinal associations between functional abilities, cognitive status, and depressive symptoms in AD. 517 patients diagnosed with probable AD and enrolled in The Multicenter Study of Predictors of Disease Course in Alzheimer's Disease were included. Patients were followed at 6-month intervals over 5.5 years. Longitudinal changes in the Blessed Dementia Rating Scale, modified Mini-Mental State Exam, and the depression subscale of the Columbia University Scale for Psychopathology in AD were examined in a multivariate latent growth curve model that controlled for gender, age, education, and recruitment site. Results showed that cognition and function worsened over the study period, whereas depressive symptoms were largely stable. Rates of change in cognition and function were correlated across participants and coupled within participants, indicating that they travel together over time. Worse initial cognitive status was associated with faster subsequent functional decline, and vice versa. Higher level of depressive symptoms was associated with worse initial functioning and faster subsequent cognitive and functional decline. These findings highlight the importance of both cognitive and psychiatric assessment for functional prognosis. Targeting both cognitive and depressive symptoms in the clinical treatment of AD may have incremental benefit on functional abilities.
\end{abstract}

Keywords: Activities of daily living, Alzheimer's disease, depression, statistical models

\section{INTRODUCTION}

Alzheimer's disease $(\mathrm{AD})$ is an age-related neurodegenerative disorder characterized by declines in both cognitive skills and functional abilities. Despite an intuitive link between cognition and function, these variables are only moderately correlated in crosssectional analyses [1], and longitudinal studies of their association in patients with $\mathrm{AD}$ are less common.

\footnotetext{
${ }^{*}$ Correspondence to: Yaakov Stern, Sergievsky Center/Taub Institute, Columbia University, 630 168th Street, P \& S Box 16, New York, NY 10032, USA. Tel.: +1 212342 1350; Fax: +1 212 342 1838; E-mail: ys11@columbia.edu.
}

Understanding the relationship between cognition and function over the course of AD has important implications in terms of choosing outcomes in clinical trials, predicting real-world function based on cognitive data, and ascertaining clinical prognosis.

One potential non-cognitive contributor to functional status is depression, which is associated with increased risk of developing $\mathrm{AD}[2,3]$. Among individuals already diagnosed with $\mathrm{AD}$, depression occurs in up to $50 \%$ of patients and has been cross-sectionally associated with worse functional abilities, independent of cognitive status $[4,5]$. Depression may also predict future cognitive decline in $\mathrm{AD}[6]$. The influence of 
depression on the clinical course of AD is uncertain and may differ between individuals. Thus, there is a need for large, longitudinal studies of depressive symptoms, cognition, and function in AD.

A recent population-based study of $328 \mathrm{AD}$ patients reported that rates of change in function correlated with rates of change in cognitive and neuropsychiatric symptoms over 3.8 years [7]. However, this study only examined between-person associations between rates of change, and the use of mixed effects models precluded an examination of multiple trajectories within a single, controlled model. Further, only global neuropsychiatric functioning was measured rather than specific symptoms (e.g., depressive) despite evidence that different neuropsychiatric symptom clusters exhibit distinct trajectories in AD [8].

Previous studies from our group using data from the Multicenter Study of Predictors of Disease Course in Alzheimer's Disease ("Predictors Study") [9, 10] have shown that compared to psychosis and behavioral disturbance, depressive symptoms do not commonly appear de novo during the course of $\mathrm{AD}$ [11]. Further, depressive symptoms decrease with $\mathrm{AD}$ progression [12]. The present study aimed to extend these findings to an examination of the influence of depressive symptoms on functional ability, an important clinical outcome. Specifically, we examined the trajectories of functional, cognitive, and depressive symptoms in a large sample of $\mathrm{AD}$ patients followed over 5.5 years using multivariate latent growth curve modeling.

\section{MATERIAL AND METHODS}

\section{Participants and procedures}

The present sample included 517 patients diagnosed with probable $\mathrm{AD}$ and enrolled in the Multicenter Study of Predictors of Disease Course in Alzheimer's Disease. Local Institutional Review Boards at all participating sites approved the study. Full study procedures are described elsewhere [9, 10]. In brief, patients were recruited in two waves at outpatient clinics and clinical research centers at four sites in the United States and Europe: Columbia University Medical Center $(n=208)$, Johns Hopkins School of Medicine $(n=147)$, Massachusetts General Hospital $(n=124)$, and the Hôpital de la Salpêtrière in Paris, France $(n=38)$. Diagnoses of probable AD were made using NINCDS-ADRDA criteria [13] at consensus conferences attended by at least two faculty physicians specializing in dementia and one faculty neuropsychologist. Complete inclusion and exclusion criteria for the study have been described previously $[9,10]$. All patients were required to have mild dementia, as defined by a Modified Mini-Mental State Exam (mMMS) score $\geq 30$ (see description of this measure below), which is equivalent to a Folstein Mini-Mental State Exam score $\geq 16$. Exclusion criteria were evidence for a cause of dementia other than Alzheimer's disease, parkinsonism, stroke, alcoholism, schizophrenia, schizoaffective disorder, and electroconvulsive therapy within two years preceding study enrollment or a history of 10 or more electroconvulsive treatments in a single course.

Average age was $74.19(\mathrm{SD}=8.45)$. Average level of education was 13.72 (3.62). The sample comprised $43.1 \%$ males, $93.2 \%$ Caucasians, and $95.2 \%$ nonHispanics. 182 participants were reported to have taken a prescription medication for dementia at some point during the 5.5-year study period. The vast majority $(n=176)$ of participants taking an anti-dementia medication were recruited in the second wave (i.e., after 1997). Only six of the 252 participants recruited before 1997 were reported to have taken an anti-dementia medication. 166 participants were reported to have taken an antidepressant at some point during the study period.

Participants were assessed prospectively at 6-month intervals for up to 16 years. At least one follow-up assessment was available for $96.52 \%$ of the present sample. The average number of assessments was 10.10 ( standard deviation $=5.85$ ), indicating that the average participant was followed semiannually over 5 years. Only data from the first 12 occasions (5.5 years) were included in the present study in order to maximize covariance coverage. Information on outcome measures across the 12 occasions is provided in Table 1. 193 participants were assessed with at least one outcome measure at the 12 th occasion. These participants did not differ from the 324 participants who were not assessed at the 12th occasion in terms of age, education, race, ethnicity, or depressive symptoms. However, they exhibited lower cognition and functioning at baseline, as assessed by the mMMS (36.91 versus $38.84 ; t(512)=-3.364 ; p<0.001)$ and the Blessed Dementia Rating Scale (BDRS; 3.80 versus 2.86; $t(435.695)=5.296 ; p<0.001)$, respectively.

\section{Measures}

Depressive symptoms were assessed at each occasion with the Columbia University Scale for 
Table 1

Patient scores at each visit

\begin{tabular}{|c|c|c|c|c|c|c|c|c|c|}
\hline \multirow[b]{2}{*}{ Year } & \multicolumn{3}{|c|}{ BDRS } & \multicolumn{3}{|c|}{ mMMS } & \multicolumn{3}{|c|}{ CUSPAD depressed mood } \\
\hline & $n$ & Mean & $\mathrm{SD}$ & $n$ & Mean & SD & $n$ & Mean & $\mathrm{SD}$ \\
\hline 0 & 507 & 3.44 & 2.05 & 514 & 37.63 & 6.37 & 509 & 0.74 & 1.05 \\
\hline 0.5 & 452 & 4.10 & 2.45 & 445 & 35.38 & 8.54 & 428 & 0.67 & 0.97 \\
\hline 1 & 427 & 4.97 & 2.99 & 421 & 33.04 & 10.11 & 399 & 0.80 & 1.05 \\
\hline 1.5 & 394 & 5.81 & 3.46 & 377 & 30.81 & 10.88 & 366 & 0.77 & 1.06 \\
\hline 2 & 361 & 6.31 & 3.61 & 345 & 29.39 & 12.00 & 339 & 0.70 & 1.00 \\
\hline 2.5 & 346 & 7.14 & 3.92 & 312 & 27.33 & 12.86 & 326 & 0.69 & 1.04 \\
\hline 3 & 310 & 7.67 & 4.08 & 277 & 25.61 & 14.20 & 286 & 0.64 & 0.96 \\
\hline 3.5 & 275 & 8.26 & 3.95 & 239 & 24.64 & 13.77 & 258 & 0.66 & 0.95 \\
\hline 4 & 266 & 8.68 & 4.15 & 225 & 24.05 & 14.18 & 235 & 0.60 & 0.97 \\
\hline 4.5 & 214 & 9.41 & 4.25 & 178 & 21.96 & 15.06 & 190 & 0.61 & 0.95 \\
\hline 5 & 206 & 9.66 & 4.33 & 166 & 22.95 & 14.89 & 182 & 0.58 & 0.94 \\
\hline 5.5 & 191 & 10.28 & 4.31 & 136 & 22.21 & 13.96 & 167 & 0.69 & 1.10 \\
\hline
\end{tabular}

57); CUSPAD, Columbia Scale for Psychopathology in Alzheimer's Disease (range: 0 to 4).

Psychopathology in AD (CUSPAD) [14]. The CUSPAD is a semi-structured interview conducted with an informant assessing the presence and severity of delusions, hallucinations, illusions, behavioral disturbances, and depressive symptoms over the past month. Only depressive symptom severity scores were used in the present study. Scores range from 0 to 4 , with higher scores indicating greater psychopathology. Good inter-rater reliability for concurrent ratings of the depressive symptoms portion of a single interview has been reported $(\kappa=0.80)$ [14]. Separate models treating the depressive symptoms variable as continuous versus categorical were run. Importantly, there were no differences in the patterns of associations between latent variables in these models. Because a larger number of fit indices are provided in the continuous variable models, results from these models are presented.

Global cognitive status was evaluated at each occasion with the mMMS [15]. In addition to items from the Mini-Mental State Exam [16], the mMMS includes items allowing for more comprehensive assessment of working memory, calculation, recall of the current and four previous presidents of the United States, confrontation naming, repetition, and visuoconstruction. The scale was translated and modified for assessments at the Paris site. Scores range from 0 to 57, with higher scores indicating better cognitive functioning.

Functional abilities were assessed with the BDRS [17]. The BDRS is a semi-structured interview conducted with an informant that assesses a patient's difficulty performing various activities of daily living for non-physical reasons. The sum of items specifically assessing instrumental (1-7) and basic (9-11) activities of daily living were used in the present study. Scores range from 0 to 16 , with higher scores indicating greater functional difficulty.

\section{Statistical analysis}

Data were analyzed in MPlus version 7 with a special case of structural equation modeling often referred to as latent growth curve (LGC) modeling using maximum likelihood estimation $[18,19]$. Missing data were managed with full information maximum likelihood (FIML), which uses all available data for parameter estimation. This approach accumulates and maximizes casewise likelihood functions computed using all available data for each participant. Monte Carlo simulation has shown that FIML produces unbiased and more efficient estimates than alternative methods (e.g., listwise deletion, pairwise deletion, and similar response pattern imputation) [20]. Models carry the assumptions of homogeneity of error variance and dependence of errors within each domain (i.e., function, cognition, depressive symptoms). A strength of LGC modeling is that it allows the study of multiple outcomes over time in a multivariate framework. The overall level of (intercept) and amount of change in (slope) each symptom type (i.e., functional, cognitive, depressive) represented the key parameters. Additional information regarding parameter estimation in multivariate LGC and its application to the study of neurodegenerative disease are available elsewhere [18, 21]. Model fit was assessed with the following, commonly-used statistics: Chi square, Akaike information criterion (AIC), sample size-adjusted Bayesian information criterion (BIC), root mean square error of approximation (RMSEA), comparative fit index (CFI), and TuckerLewis index (TLI). Smaller values of chi square, AIC, BIC, and RMSEA indicate better model fit. Values of CFI and TLI that are closer to 1 indicate better fit. Fit between nested models was compared statistically using the chi square test. 
Model building proceeded in two broad stages. First, the trajectories of the three variables of interest were examined separately with unconditional univariate growth curve models. To characterize the functional forms of functional, cognitive, and depressive trajectories, models that estimated only linear change were statistically compared to those that estimated both linear and quadratic change. Second, best-fitting univariate models were combined into a single, conditional multivariate model, in which obtained parameter estimates control for all included variables. In this model, correlations between initial levels and changes in the symptoms independent of the covariates (i.e., gender, age, education in years, and recruitment site) can be estimated. Within-person coupling of symptoms over time was examined via correlations between occasion-specific residuals [22]. These correlations reflect the relationship between state-like, occasionspecific changes in the different symptom domains after controlling for a person's trait-like change trajectories. For parsimony, correlations of occasion-specific residuals were constrained to be equal across occasions for each symptom domain pair. In addition, covariate effects on both the overall levels of symptoms (intercepts) as well as on symptom changes (slopes) were examined. Covariates were centered to facilitate parameter interpretation. Specifically, values of 0 corresponded to age 74, 12 years of education, male gender, and enrollment at the Columbia site.

\section{RESULTS}

\section{Unconditional univariate models}

Nested unconditional univariate models were built separately for the three outcome variables.
In models allowing only linear change, functional problems (slope estimate $=1.150 ; p<0.001$ ) and cognitive symptoms (slope estimate $=-4.783 ; p<0.001$ ) worsened over the study period. In contrast, depressive symptoms improved (slope estimate $=-0.024$; $p=0.043$ ). Allowing for curvilinear change significantly improved model fit for functional problems $\left(\Delta \chi^{2}(4)=-438.77, p<0.001\right)$, cognitive symptoms $\left(\Delta \chi^{2}(4)=-503.18, p<0.001\right)$, and depressive symptoms $\left(\Delta \chi^{2}(4)=-34.66, p<0.001\right)$. Thus, models including both linear and quadratic slopes were retained for subsequent model building.

Parameter estimates in the best-fitting unconditional univariate LGC models are shown in the upper panel of Table 2. Intercepts (levels) refer to latent variables derived from all 12 occasions that reflect estimated initial levels of the outcomes independent of the growth process, not merely baseline scores. Linear slopes can be interpreted as the constant rates of change over time. Quadratic slopes can be interpreted as changes in the rates of change over time. As shown in Fig.1, functional problems and cognitive symptoms worsened over the study period. This worsening of cognitive symptoms was most dramatic early on and leveled out over time. Although the model of depressive symptoms allowing for curvilinear change fit the data better than the model allowing only linear change, neither slopes reached significance in the more complex model, indicating that depressive symptoms were largely stable among the sample as a whole. However, significant residual variances in both intercepts and slopes (all $p ' s<0.001$ ) in all three models indicate substantial individual differences both in initial levels and trajectories of functional abilities, cognition, and depressive symptoms. Such residual variance is a precondition for further model building.

Table 2

Unstandardized parameter estimates

\begin{tabular}{|c|c|c|c|c|c|c|}
\hline & \multicolumn{2}{|c|}{ Intercept (level) } & \multicolumn{2}{|c|}{ Linear slope } & \multicolumn{2}{|c|}{ Quadratic slope } \\
\hline & Intercept (SE) & Variance (SE) & Intercept (SE) & Variance (SE) & Intercept (SE) & Variance (SE) \\
\hline \multicolumn{7}{|c|}{ Unconditional univariate $^{\mathrm{a}}$} \\
\hline BDRS & $3.430(0.093) * *$ & $3.783(0.283) * *$ & $1.679(0.103) * *$ & $4.054(0.339)^{* *}$ & $-0.024(0.019)$ & $0.112(0.012)^{* *}$ \\
\hline mMMS & $37.699(0.284)^{* *}$ & $37.874(2.617)^{* *}$ & $-5.181(0.296) * *$ & $34.209(2.808) * *$ & $0.159(0.057)^{*}$ & $0.947(0.105)^{* *}$ \\
\hline CUSPAD & $0.771(0.044)^{* *}$ & $0.505(0.070)^{* *}$ & $0.012(0.036)$ & $0.151(0.042)^{* *}$ & $-0.007(0.007)$ & $0.006(0.002)^{* *}$ \\
\hline \multicolumn{7}{|c|}{ Conditional multivariate } \\
\hline BDRS & $3.621(0.191)^{* *}$ & $3.219(0.279)^{* *}$ & $1.461(0.221)^{* *}$ & $4.061(0.358)^{* *}$ & $-0.014(0.041)$ & $0.110(0.012)^{* *}$ \\
\hline mMMS & $38.654(0.582)^{* *}$ & $34.045(2.583) * *$ & $-5.248(0.625)^{* *}$ & $33.808(2.914)^{* *}$ & $0.095(0.120)$ & $0.906(0.101)^{* *}$ \\
\hline CUSPAD & $0.943(0.090)^{* *}$ & $0.497(0.062)^{* *}$ & $-0.024(0.077)$ & $0.167(0.041)^{* *}$ & $-0.008(0.016)$ & $0.007(0.002)^{* *}$ \\
\hline
\end{tabular}

SE, standard error; BDRS, Blessed Dementia Rating Scale (range: 0 to 16); mMMS, modified Mini Mental State Exam (range: 0 to 57); CUSPAD, Columbia Scale for Psychopathology in Alzheimer's Disease (range: 0 to 4);

${ }^{a}$ Parameters estimated in three separate univariate models;

${ }^{*} p<0.05 ;{ }^{* *} p<0.001$. 

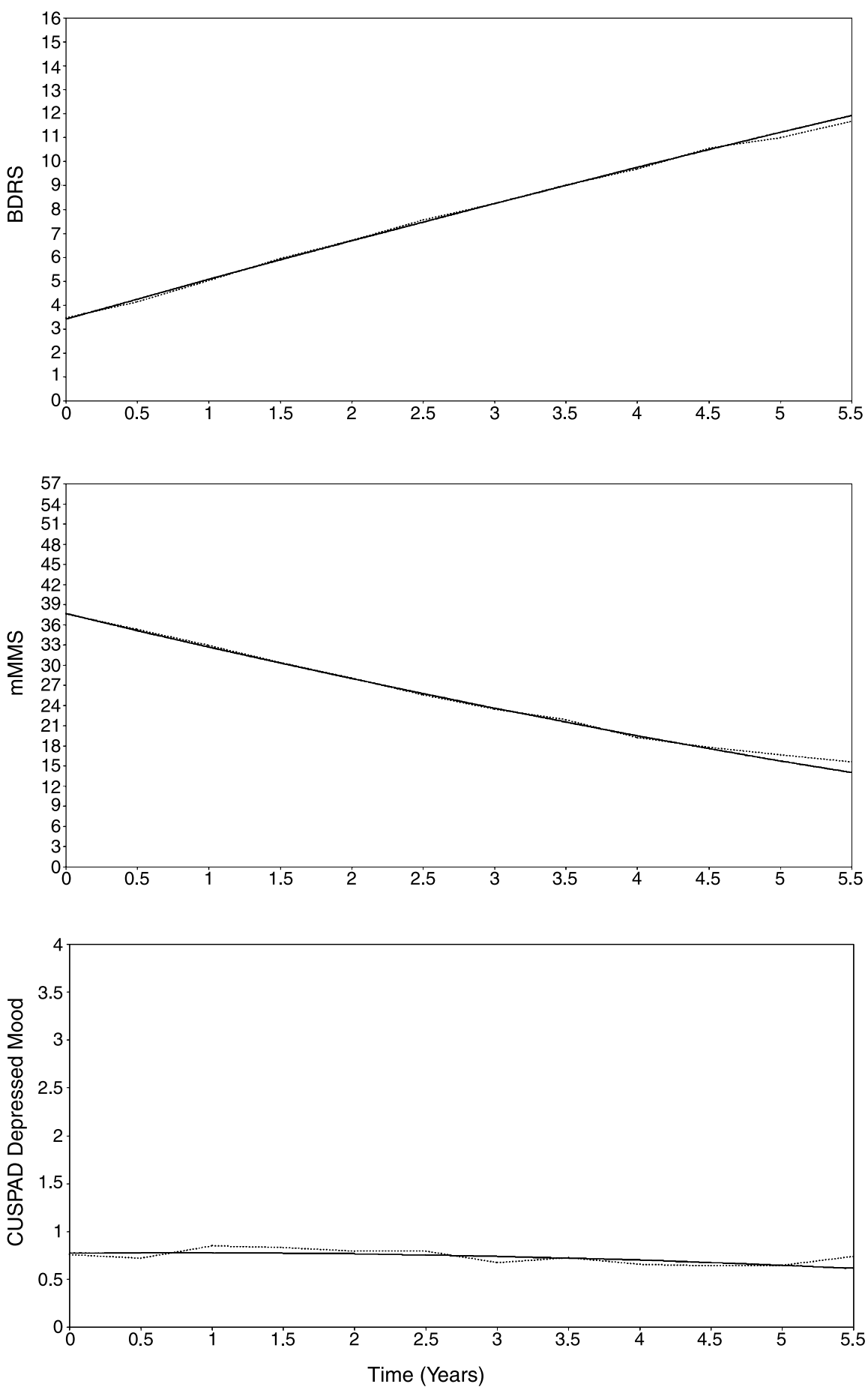

Fig. 1. Estimated growth curves from the univariate models. Dotted lines represent sample means; solid lines represent model-estimated means. Y axes display full ranges for each scale so that relative differences between symptom types can be appreciated. BDRS, Blessed Dementia Rating Scale; mMMS, modified Mini Mental State Exam; CUSPAD, Columbia Scale for Psychopathology in Alzheimer's disease.

\section{Conditional multivariate model}

The three best-fitting univariate LGC models were combined into a single multivariate model, and four time-invariant predictors measured at baseline (i.e., age, gender, education, and recruitment site) were added. First, a transition model in which 
neither cross-domain relationships between latent variables nor associations involving the covariates was estimated. In line with the univariate models, associations between latent variables within a domain were permitted. The transition model provided the following fit statistics: $\chi^{2}(788)=2052.182(p<0.001)$; $\mathrm{AIC}=57513.757 ; \quad \mathrm{BIC}=57819.616 ; \quad \mathrm{CFI}=0.894 ;$ $\mathrm{TLI}=0.895$ and RMSEA $=0.056 \quad(0.053,0.059)$. Figure 2 presents a schematic representation of the conditional multivariate model, which estimated all possible regression paths and covariances. Compared to the transition model, fit significantly improved $\left(\Delta \chi^{2}(38)=-516.975, \quad p<0.001\right)$. The model provided the following fit statistics: $\chi^{2}(750)=1535.207$ $(p<0.001) ; \quad$ AIC $=48315.510 ; \quad$ BIC $=48723.136$; $\mathrm{CFI}=0.934 ; \mathrm{TLI}=0.932$ and $\mathrm{RMSEA}=0.045(0.042$, 0.048).

Intercepts shown in the lower panel of Table 2 can be interpreted as estimated initial values when all covariates are set to 0 (i.e., age $=74$; education $=12$ years; gender $=$ male; site $=$ Columbia $)$. As shown, worsening over time was evident for functional problems and cognitive symptoms after controlling for these variables. Changes in depressive symptoms over time were not significant.

Associations between the factors in the conditional model are shown in Table 3. After controlling for the covariates, initial levels predicted subsequent changes within cognition and function domains. Independent of these associations, initial levels of functional problems and cognitive symptoms were correlated. Worse initial cognitive status was also associated with faster subsequent functional decline, and vice versa. Rates of decline in functional and cognitive status were correlated between individuals (see Table 3) as well as coupled within individuals (standardized estimate $=-0.207 ;$ S.E. $=0.021 ; p<0.001)$. Higher level of depressive symptoms was associated with worse initial functioning and faster subsequent cognitive and functional decline.

Older age was independently associated with greater initial functional disability (standardized parameter estimate $=0.28 ; \mathrm{SE}=0.05 ; p=0.001$ ) but slower cognitive decline (standardized parameter estimate $=0.25$; $\mathrm{SE}=0.05 ; p=0.001)$ and less worsening of depressive symptoms. Female gender was associated only with lower initial mMMS scores (standardized parameter estimate $=-0.16 ; \mathrm{SE}=0.05 ; p=0.001$ ). Lower level of education was associated with lower cognitive status at baseline (standardized parameter estimate $=0.21$; $\mathrm{SE}=0.05 ; p<0.001)$ but not with the average rate of cognitive decline. Participants enrolled at different recruitment sites differed only in initial depressive symptoms.

\section{Influence of attrition and medication use}

In order to determine whether attrition influenced the results, a variable reflecting the participation in the 12th assessment was added to the multivariate model. In addition to worse initial levels of cognition and function, participants who did not complete all 5.5 years of follow-up exhibited accelerated cognitive decline (standardized parameter estimate $=-0.238$; $p<0.001$ ) and more rapid increases in functional problems (standardized parameter estimate $=0.186$; $p<0.001)$. Importantly, inclusion of this variable did not alter the pattern of associations summarized in Table 3.

The potential influence of anti-dementia medications was investigated in the subset of 265 participants recruited after 1997 because only six of the 252 participants recruited during the earlier wave took a prescription medication for dementia. A variable reflecting use of anti-dementia medication at any point during the 5.5-year study period was added to the three univariate models for function, cognition, and depressive symptoms. Anti-dementia medication use was not associated with initial levels or rates of change for depressive symptoms or cognition. Anti-dementia medication use was associated with lower initial levels of functional problems (unstandardized parameter estimate $=-0.78 ; p=0.01$ ), but not with rates of change in functioning. Thus, anti-dementia medication use did not appear to influence the trajectories of any outcomes of interest.

In order to determine whether antidepressant use influenced the results, a variable reflecting use of an antidepressant at any point during the 5.5-year study period was added to the multivariate model estimated in the full sample. Antidepressant use was associated with higher initial levels of depressive symptoms (unstandardized parameter estimate $=0.35$; $p<0.001$ ). In addition, antidepressant use was associated with better initial cognitive (unstandardized parameter estimate $=1.45 ; p=0.02$ ) and functional status (unstandardized parameter estimate $=-0.42$; $p=0.04$ ). Importantly, antidepressant use was not associated with any of the symptom trajectories, and inclusion of this additional covariate did not alter the pattern of results described above. 


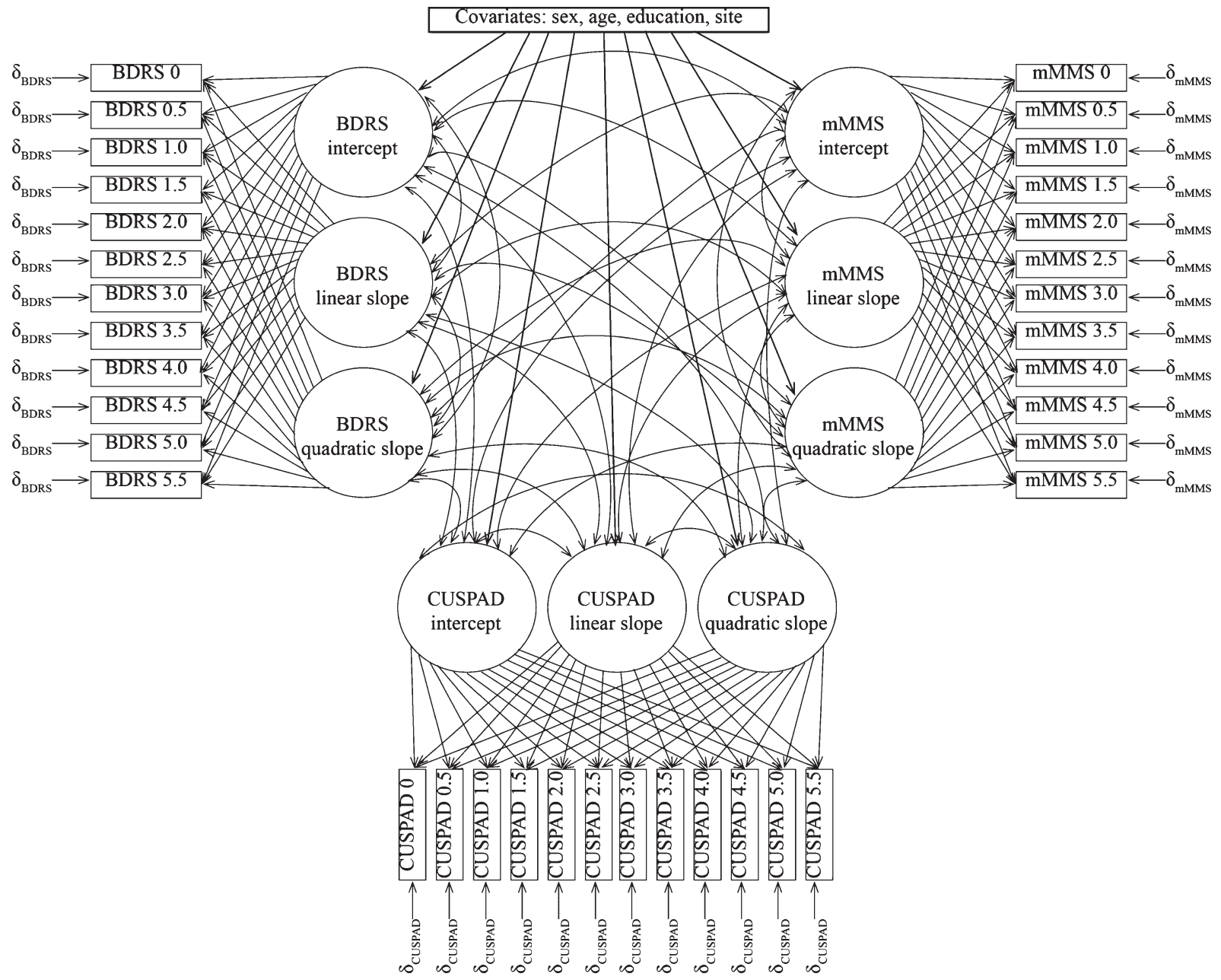

Fig. 2. Schematic representation of the conditional multivariate latent growth curve model. BDRS, Blessed Dementia Rating Scale; mMMS, modified Mini Mental State Exam; CUSPAD, Columbia Scale for Psychopathology in Alzheimer's disease.

\section{DISCUSSION}

This longitudinal study of 517 individuals with AD replicated previous reports from our group that while functional and cognitive symptoms gradually worsen over the disease course, depressive symptoms do not [12]. The main findings from the multivariate latent growth curve analysis were: 1) cognitive and functional changes were correlated between individuals and coupled within individuals; 2) worse initial cognitive status predicted faster subsequent functional decline, and vice versa; and 3) depressive symptoms were associated with worse initial functioning and faster subsequent cognitive and functional decline.

Our finding of a significant longitudinal association between functional and cognitive changes in
AD confirms and extends a previous report [7] by demonstrating that these outcomes are associated both between and within persons over time in a controlled, multivariate model. These results also support the use of the mMMS [15], a measure of global cognitive status, as a gross primary outcome in clinical trials and studies of AD course. Indeed, this measure appears to exhibit a similar rate of change as an informantrated measure of performance ability and therefore can adequately track the longitudinal course of functional decline in AD.

The obtained between-persons association between rates of functional and cognitive changes in our multivariate model (standardized parameter estimate $=-0.72)$ is very similar to that reported in a population-based study of $\mathrm{AD}(r=-0.62)$ [7]. 
Table 3

Standardized between-persons associations between the latent factors in the conditional multivariate model

\begin{tabular}{|c|c|c|c|c|c|c|c|c|c|}
\hline & \multicolumn{3}{|c|}{ BDRS } & \multicolumn{3}{|c|}{ mMMS } & \multicolumn{3}{|c|}{ CUSPAD } \\
\hline & Intercept & Linear & Quadratic & Intercept & Linear & Quadratic & Intercept & Linear & Quadratic \\
\hline \multicolumn{10}{|l|}{ BDRS } \\
\hline Intercept & - & & & & & & & & \\
\hline Linear & $0.138^{*}$ & - & & & & & & & \\
\hline Quadratic & $-0.227 *$ & $-0.894 * *$ & - & & & & & & \\
\hline \multicolumn{10}{|l|}{ mMMS } \\
\hline Intercept & $-0.437 * *$ & $-0.345^{* *}$ & $0.321 * *$ & - & & & & & \\
\hline Linear & $-0.203^{*}$ & $-0.719 * *$ & $0.536^{* *}$ & $0.417 * *$ & - & & & & \\
\hline Quadratic & $0.235^{*}$ & $0.631 * *$ & $-0.618 * *$ & $-0.471 * *$ & $-0.854 * *$ & - & & & \\
\hline \multicolumn{10}{|l|}{ CUSPAD } \\
\hline Intercept & $0.208^{*}$ & $0.174^{*}$ & -0.158 & -0.086 & $-0.146^{*}$ & $0.197 *$ & - & & \\
\hline Linear & -0.108 & 0.039 & -0.022 & 0.026 & 0.073 & -0.154 & -0.157 & - & \\
\hline Quadratic & -0.009 & -0.149 & 0.137 & 0.048 & 0.055 & 0.029 & -0.068 & $-0.938 * *$ & - \\
\hline
\end{tabular}

BDRS, Blessed Dementia Rating Scale; mMMS, modified Mini Mental State Exam; CUSPAD, Columbia Scale for Psychopathology in Alzheimer's disease.

${ }^{*} p<0.05 ;{ }^{* *} p<0.001$.

This between-person association assesses the relationship between long term trends in cognitive and functional change. In contrast, the within-person association between these outcomes in our multivariate model was more modest (standardized parameter estimate $=-0.21$ ). This within-person association assesses whether occasion-specific deviations from cognitive and functional trends occur together. This finding indicates that the rate at which an individual patient loses the ability to perform activities of daily living is heavily influenced by factors other than cognitive decline. For example, our group has previously shown that delusions, hallucinations, extrapyramidal signs, and other motor symptoms predict functional abilities and other important outcomes (e.g., death, nursing home placement) in AD [23-26]. The present study provides evidence that depressive symptoms also have prognostic value in $\mathrm{AD}$.

Depressive symptoms were associated not only with worse functional abilities initially, but also with faster subsequent cognitive and functional decline. Many studies, though not all, have shown that depression increases dementia risk among cognitively-impaired older adults [2, 3]. Cross-sectional associations between depression and function have been reported [5], and a recent study showed an association between depression and accelerated cognitive decline in AD [6]. Fewer studies have examined the influence of depression on the functional course of patients diagnosed with AD.

We have previously shown that functional decline predicts first episodes of depressive symptoms in AD [12]. The present finding that initial depressive symptoms predicted accelerated functional decline extends this previous work by providing new evidence that the association between depressive symptoms and functioning may be bi-directional in AD. That is, the loss of functional ability may trigger a reactive depressive episode [12], and initial depressive symptoms may herald a more aggressive course of cognitive and functional decline. Thus, depressive symptoms in $\mathrm{AD}$ may not solely represent a psychological reaction to cognitive or functional decline. Similar results have also been reported in cognitively intact older adults [27].

The associations between depressive symptoms and functioning were independent of cognition. Thus, depressive symptoms appeared to negatively influence functioning directly in this cohort of $\mathrm{AD}$ patients. Depressive symptoms were also independently related to cognitive decline. This pattern of findings suggests that the pathophysiologic processes leading to depressive and cognitive symptoms in $\mathrm{AD}$ may be partially overlapping. In previous studies, depressive symptoms in the context of dementia have been linked to pathology within the brain stem and substanta nigra and to reduced cortical norepinephrine [28, 29].

A limitation of this study is the inclusion of only a subset of variables associated with cognition and function due to modeling constraints. The included covariates were chosen based on the extant literature as well as on significant univariate associations with the outcome variables in this sample. Other variables have been examined in previous reports from our group. For example, we have previously shown that APOE genotype is not associated with depressive symptoms 
in this cohort [30]. Another limitation is our use of a single screening measure to assess cognitive symptoms (i.e., the mMMS). A more complete cognitive battery would have allowed for exploration of how depressive symptoms influence changes in different cognitive domains. For example, it is possible that the association between initial depressive symptoms and functional decline may reflect executive deficits. Indeed, executive functioning is not fully assessed by the mMMS. It should also be noted that compared to participants assessed at the 5.5-year visit, those who were not assessed at this occasion exhibited worse cognitive and functional status at baseline and accelerated cognitive and functional decline. However, inclusion of a variable reflecting drop-out did not alter the pattern of results.

Strengths of this study include its use of latent growth curve modeling, as the examination of both initial levels and trajectories of symptoms provided more comprehensive evidence regarding their longitudinal associations. Another major strength is the modeling of three outcome variables in a single model, which allowed for more precise estimates of unique associations and improved upon previous studies in which models contained single outcome variables.

In conclusion, this study provides evidence that cognitive and functional symptoms travel together in $\mathrm{AD}$. Results also suggest that cognitive decline predicts later functional decline, highlighting the importance of cognitive assessments for functional prognosis. Finally, disease progression appears to be heavily influenced by non-cognitive factors, as depressive symptoms predicted accelerated cognitive and functional decline independently.

\section{ACKNOWLEDGMENTS}

The Predictors study is supported by NIA R01 AG007370 to Dr. Stern, Dr. Zahodne is supported by NIA T32 AG000261. This publication was also supported by the National Center for Advancing Translational Sciences, National Institutes of Health, through Grant Number UL1 TR000040, formerly the National Center for Research Resources, Grant Number UL1 RR024156. The content is solely the responsibility of the authors and does not necessarily represent the official views of the NIH. The sponsors had no role in the study design or data interpretation.

Authors' disclosures available online (http://www. j-alz.com/disclosures/view.php?id=1606).

\section{REFERENCES}

[1] Feldman HH, Baelen BV, Kavanagh SM, Torfs KE (2005) Cognition, function, and caregiving time patterns in patients with mild-to-moderate Alzheimer disease: A 12-month analysis. Alzheimer Dis Assoc Disord 19, 29-36.

[2] Devanand DP, Sano M, Tang MX, Taylor S, Gurland BJ, Wilder D, Stern Y, Mayeux R (1996) Depressed mood and the incidence of Alzheimer's disease in the elderly living in the community. Arch Gen Psychiatr 53, 175-182.

[3] Gao Y, Huang C, Zhao K, Ma L, Qiu X, Zhang L, Xiu Y, Chen L, Lu W, Huang C, Tang Y, Xiao Q (2012) Depression as a risk factor for dementia and mild cognitive impairment: A meta-analysis of longitudinal studies. Int J Geriatr Psychiatr, doi: $10.1002 /$ gps.3845

[4] Starkstein SE, Jorge R, Mizrahi R, Robinson RG (2005) The construct of minor and major depression in Alzheimer's disease. Am J Psychiatry 162, 2086-2093.

[5] Pearson JL, Teri L, Reifler BV, Raskind MA (1989) Functional status and cognitive impairment in Alzheimer's patients with and without depression. J Am Geriatr Soc 37, 1117-1121.

[6] Spalletta G, Caltagirone C, Girardi P, Gianni W, Casini AR, Palmer K (2012) The role of persistent and incident major depression on rate of cognitive deterioration in newly diagnosed Alzheimer's disease patients. Psychiatry Res 198, 263-268.

[7] Tschanz JT, Corcoran CD, Schwartz S, Treiber K, Green RC, Norton MC, Mielke MM, Piercy K, Steinberg M, Rabins PV, Leoutsakos JM, Welsh-Bohmer KA, Breitner JC, Lyketsos CG (2011) Progression of cognitive, functional, and neuropsychiatric symptom domains in a population cohort with Alzheimer dementia: The Cache County Dementia Progression Study. Am J Geriatr Psychiatry 19, 532-542.

[8] Garre-Olmo J, López-Pousa S, Vilalta-Franch J, de Gracia Blanco M, Vilarrasa AB (2010) Grouping and trajectories of neurosychiatric symptoms in patients with Alzheimer's disease. Part II: Two-year patient trajectories. J Alzheimers Dis 22, 1169-1180.

[9] Stern Y, Folstein M, Albert M, Richards M, Miller L, Bylsma F, Lafleche G, Marder K, Bell K, Sano M, et al. (1993) Multicenter study of predictors of disease course in Alzheimer's disease (the "Predictors Study"): I Study design, cohort description and intersite comparisons. Alzheimer Dis Assoc Disord 7, 3-21.

[10] Richards M, Folstein M, Albert M, Miller L, Bylsma F, Lacfleche G, Marder K, Bell K, Sano M, Devanand D, et al. (1993) Multi-center study of predictors of disease course in Alzheimer's disease (the 'Predictors Study') II. Baseline findings. Alzheimer Dis Assoc Disord 7, 22-32.

[11] Devanand DP, Jacobs DM, Tang MX, Del Castillo-Castaneda C, Sano M, Marder K, Belle K, Bylsma FW, Brandt J, Albert M, Stern Y (1997) The course of psychopathologic features in mild to moderate Alzheimer disease. Arch Gen Psychiatr 54, 257-263.

[12] Holtzer R, Scarmeas N, Wegesin DJ, Albert M, Brandt J, Dubois B, Hadjigeorgioiu GM, Stern Y (2005) Depressive symptoms in Alzheimer's disease: Natural course and temporal relation to function and cognitive status. J Am Geriatr Soc 53, 2083-2089.

[13] McKhann G, Drachman D, Folstein M, Katzman R, Price D, Stadlan EM (1984) Clinical diagnosis of Alzheimer's disease. Report of the NINCDS-ADRDA Work Group under the auspices of the Department of Health and Human Services Task Force on Alzheimer's disease. Neurology 34, 939-944. 
[14] Devanand DP, Miller L, Richards M, Marder K, Belle K, Mayeux R, Stern Y (1992) The Columbia University Scale for Psychopathology in Alzheimer's disease. Arch Neurol 49, 371-376.

[15] Stern Y, Sano M, Paulson J, Mayeux R (1987) Modified minimental state examination: Validity and reliability. Neurology 37(Suppl. 1), 179.

[16] Folstein MF, Folstein SE, McHugh PR (1975) 'Mini-mental state'. A practical method for grading the cognitive state of patients for the clinician. J Psychiatr Res 12, 189-198.

[17] Blessed G, Tomlinson BE, Roth M (1968) The association between quantitative measures of senile change in the cerebral grey matter of elderly subjects. Br J Psychol 114, 797-811.

[18] Bollen KA, Curran PJ (2006) Latent Curve Models: A Structural Equation Perspective. Wiley, Hoboken.

[19] Muthen LK, Muthen BO (1998) Mplus User's Guide. Muthen \& Muthen, Los Angeles.

[20] Enders CK, Bandalos DL (2001) The relative performance of full information maximum likelihood estimation for missing data in structural equation models. Struct Equation Model 8 , 430-457.

[21] Zahodne LB, Marsiske M, Okun MS, Rodriguez RL, Malaty I, Bowers D (2012) Mood and motor trajectories in Parkinson's disease: Multivariate latent growth curve modeling. Neuropsychology 26, 71-80.

[22] Hofer SM, Gray KM, Piccinin AM, MacKinnon A, Bontempo DE, Einfeld SL, Hoffman L, Parmenter T, Tonge BJ (2009) Correlated and coupled within-person change in emotional and behavior disturbance in individuals with intellectual disability. Am J Intellect Dev Disabil 114, 305-321.

[23] Stern Y, Albert M, Brandt J, Jacobs DM, Tang MX, Marder K, Bell K, Sano M, Devanand DP, Bylsma F, et al. (1994) Utility of extrapyramidal signs and psychosis as predictors of cognitive and functional decline, nursing home admission and death in Alzheimer's disease: Prospective analyses from the "Predictors Study". Neurology 44, 2300-2307.

[24] Stern Y, Liu X, Albert M, Brandt J, Jacobs DM, Del CastilloCastaneda C, Marder K, Bell K, Sano M, Bylsma F (1996) Modeling the influence of extrapyramidal signs on the progression of Alzheimer's disease. Arch Neurol 53, 11211126.

[25] Scarmeas N, Albert M, Brandt J, Blacker D, Hadjigeorgiou G, Papadimitriou A, Dubois B, Sarazin M, Wegesin D, Marder K, Bell K, Honig L, Stern Y (2005) Motor signs predict poor outcomes in Alzheimer's disease. Neurology 64, 1696-1703.

[26] Scarmeas N, Brandt J, Albert M, Hadjigeorgiou G, Papadimitriou A, Dubois B, Sarazin M, Devanand D, Honig L, Marder K, Bell K, Wegesin D, Blacker D, Stern Y (2005) Delusions and hallucinations are associated with worse outcome in Alzheimer disease. Arch Neurol 62, 1601-1608.

[27] Panza F, D'Introno A, Colacicco AM, Capurso C, Del Parigi A, Caselli RJ, Frisardi V, Scapicchio P, Chiloiro R, Scafato E, Gandin C, Vendemiale G, Capurso A, Solfrizzi V (2009) Temporal relationship between depressive symptoms and cognitive impairment: The Italian Longitudinal Study on Aging. J Alzheimers Dis 17, 899-911.

[28] Zubenko GS, Moossy J (1988) Major depression in primary dementia: Clinical and neuropathologic correlates. Arch Neurol 45, 1182-1186.

[29] Zubenko FS, Moossy J, Kopp U (1990) Neurochemical correlates of major depression in primary dementia. Arch Neurol 47, 209-214.

[30] Scarmeas N, Brandt J, Albert M, Devanand DP, Marder K, Bell K, Ciappa A, Tycko B, Stern Y (2002) Association between the APOE genotype and psychopathologic symptoms in Alzheimer's disease. Neurology 58, 1182-1188. 